\title{
Fission Product Decontamination Factors for Plutonium Separated by PUREX from Low-Burnup, Fast-Neutron Irradiated Depleted $\mathrm{UO}_{2}$
}

\author{
Paul M. Mendoza a,b, Sunil S. Chirayatha,b, ${ }^{a,}$ Charles M. Folden IIIc \\ aNuclear Security Science \& Policy Institute, Texas A\&M University, College Station, TX 77843 \\ USA \\ bDepartment of Nuclear Engineering, Texas A\&M University, College Station, TX 77843 USA \\ 'Cyclotron Institute, Texas A\&M University, College Station, TX 77843 USA
}

\begin{abstract}
Experimental investigations to determine fission product separation from actinides $(\mathrm{U}$ and $\mathrm{Pu})$ while employing the Plutonium Uranium Recovery by Extraction (PUREX) process to purify plutonium produced in a fast neutron irradiated depleted uranium dioxide $\left(\mathrm{DUO}_{2}\right)$ target were conducted. The sample was a $\mathrm{DUO}_{2}$ pellet $\left(0.256 \mathrm{wt} \%{ }^{235} \mathrm{U}\right)$ irradiated to a low-burnup (4.43 \pm 0.31 $\mathrm{GWd} / \mathrm{tHM}$ ) that was PUREX processed 538 days after neutron irradiation. Decontamination factors (DF) for the elements U, Mo, Ru, Ce, Sm, Sr, Pm, Eu, $\mathrm{Nd}, \mathrm{Pd}$, and $\mathrm{Cd}$ were measured with mass spectroscopy in two experiments using 30 vol. \% tri-n-butyl phosphate (TBP) in a kerosene diluent. The first experiment characterized Pu DFs for a single stage extraction and back-extraction, while the second experiment had multiple stages with the goal of achieving greater $\mathrm{Pu}$ recovery. The benchtop scale PUREX process had overall Pu recoveries of (83.4 \pm $9.5) \%$ and $(99.7 \pm 4.2) \%$ for the first and second experiments, respectively.

Keywords: PUREX; Decontamination Factor; Irradiated Depleted Uranium
\end{abstract}

\section{Introduction}

Background. In a recent publication, our group suggested that investigation of trace contaminants in Pu recovered using the Plutonium Uranium Recovery by

*Corresponding author

Email address: sunilsc@tamu.edu (Sunil S. Chirayath)

Preprint submitted to Applied Radiation and Isotopes

August 26, 2016

C 2016. This manuscript version is made available under the Elsevier user license http://www.elsevier.com/open-access/userlicense/1.0/ 
Extraction (PUREX) process could give indications of material origins (Chirayath

5 2015). This computational study analyzed Pu and trace level contaminants of decontaminated fuel irradiated in fast breeder and heavy water thermal reactors due to their relative ease of producing weapons-grade Pu. A conclusion of the paper was that a distinction can be made between decontaminated Pu from a fast and thermal system based on fission product (FP) to Pu concentration ratios. In

10 practice, however, if a sample of $\mathrm{Pu}$ were intercepted through conventional investigative techniques, it would likely have already been processed via PUREX. Therefore, data on decontamination factors (DFs, a measure of contaminant purification), are needed for each element individually. Due to a lack of literature on DFs and distribution coefficients (DCs, the ratio of concentrations between 15 organic and aqueous phases) for the elements noted in our previous paper (Cs, $\mathrm{Sb}$, $\mathrm{Eu}, \mathrm{Rb}, \mathrm{Sr}, \mathrm{Nd}, \mathrm{Pm}$, and Sm), the study for this paper was conducted.

Descriptions of various PUREX processes are provided in many sources with explanations of chemistry including flow sheets and decontamination factors (Lanham 1950, Arker 1954, Darby 1954, Irish 1957, Stoller 1961, Benedict 1982).

20 These sources generally report overall beta or gamma DFs of up to $10^{8}$ with $\mathrm{Pu}$ recoveries of $99.7 \%$ for industrial scale reprocessing facilities. While DCs for the various process steps of PUREX have been previously reported, details about elemental DFs for PUREX cycles have been largely limited to the major activity contributors, such as ${ }^{106} \mathrm{Ru}$ and ${ }^{95} \mathrm{Zr}$ (Stoller 1961). A compilation of distribution 25 data for PUREX extraction processes provides data for $\mathrm{U}$, Th, and $\mathrm{Pu}$ in a variety of concentrations (Siddall 1957). DCs for $\mathrm{Zr}$, rare earth metals, $\mathrm{Pu}$, and Th are also 
available (Alcock 1957, Best 1957, Hesford 1957, Scargill 1957, Alcock 1958, Best 1959, Hesford 1959). Additionally, Ga has been studied for separation (Collins 2000) because it is a common contaminant in weapons-grade $\mathrm{Pu}$.

Although a DC, coupled with process information, can be used to calculate a reasonable estimate of DF (Colburn 1939, Sherwood 1952, Long 1967, Perry 2008), variability of DCs under different system conditions give rise to uncertainty in calculated results. For example, DCs between tri-n-butyl phosphate (TBP) and nitric acid $\left(\mathrm{HNO}_{3}\right)$ have been reported for $\mathrm{U}, \mathrm{Pu}, \mathrm{Zr}, \mathrm{Nb}, \mathrm{Ru}$, and the rare earth elements, but vary with $\mathrm{HNO}_{3}$ concentration and $\mathrm{U}$ saturation in TBP by up to several orders of magnitude (Sherwood 1952, Stoller 1961). These sources also derive mathematical correlations between DC and DF, but experimental PUREX DFs for a large number of individual elements were not provided.

In the current work, $12.9 \mathrm{mg}$ of depleted uranium dioxide $\left(\mathrm{DUO}_{2}\right)$ was 40 irradiated in a pseudo-fast neutron spectrum at the High Flux Isotope Reactor (HFIR) at Oak Ridge National Laboratory [described in detail in (Swinney 2014)]. The $\mathrm{DUO}_{2}$ pellet, containing FPs and $\mathrm{Pu}$, was dissolved in $\mathrm{HNO}_{3}$ and subjected to two different PUREX experiments for DF characterization and Pu product recovery. Aqueous solutions were analyzed at each step with Inductively Coupled

45 Plasma-Mass Spectrometry (ICP-MS). The experimental work used benchtop scale methods to isolate a large fraction of $\mathrm{Pu}$, measure DFs for FPs, and measure overall gamma DFs as part of a larger project to develop forensic radioanalytical capabilities at Texas A\&M University, and first results are reported here. In order to properly utilize the information presented for nuclear forensics, additional 
experiments are being conducted which will include different solution concentrations and levels of heavy metal saturation, as well as providing a more realistic simulation of an actual reprocessing plant. That additional information will provide more avenues to determine purification process steps, and will be the subject of a future publication.

55

Terminology. A DC is defined as the concentration ratio between the organic (org) and aqueous (aq) phases as shown in Equation 1, and describes the equilibrium distribution of a species in the system during PUREX separation processes (Benedict 1982):

$$
D C=\frac{c_{\text {org }}}{c_{a q}}
$$

where $c$ is the concentration of the specific species in the indicated phase. DCs are specific to an element and vary widely with the concentration and temperature of the solvents. They are also affected by saturation of $\mathrm{U}$ and $\mathrm{Pu}$ in the system and, in some cases, time since solution preparation (Stoller 1961, Simpson 2010). For

65 PUREX, the fraction of mass, $f_{\text {org }}$, deposited in the organic (TBP) phase for a single element, assuming a volume ratio between the aqueous and organic phases, $V_{R}$, is given by Equation 2 . 


$$
f_{\text {org }}=\left(1+D C^{-1} V_{R}^{-1}\right)^{-1}
$$

This is termed "extraction", where a desired solute is extracted out of an

70 aqueous solution through contact with an immiscible liquid phase. Ideally, a large fraction of desired solutes are extracted into the organic phase, while a small fraction of undesired solutes are extracted. Conversely, "back-extraction" is the process by which a desired solute is extracted out of an organic solution through contact with an immiscible aqueous solution. Both extraction and back-extraction

75 will also be termed "stage", where mass transfer occurs between two sufficiently mixed immiscible liquid phases. "Scrubbing" is the transfer of a large fraction of impurities to a second phase while having a minimal transfer of desired constituent.

After several cycles of Pu extraction/scrubbing/back-extraction are complete, 80 the measured effectiveness of a PUREX cycle is described by the DF (which is ideally determined by DCs) and measures the effectiveness with which a contaminant, $j$, is removed from a product. The product of interest in this work is $\mathrm{Pu}$, and the DF is defined by Equation 3.

$$
D F_{j}=\frac{\left.\frac{c_{j}}{c_{P u}}\right|_{\text {initial }}}{\left.\frac{c_{j}}{c_{P u}}\right|_{\text {final }}}
$$


Initial and final refer to the values before and after purification, respectively.

DFs are also characteristic of different process cycles, and may have larger values $\left(>10^{7}\right)$ for industrial scale PUREX compared to the benchtop scale version presented here because of an additional scrubbing step as well as a larger number of stages (Stoller 1961, Benedict 1982). Industrial processes often report either an

90 overall DF value, or a DF value for a single isotope.

\section{Experiment}

A commercially acquired pellet containing $12.9 \pm 0.1 \mathrm{mg}$ of $\mathrm{DUO}_{2}$ was irradiated over the course of three months with two shutdown periods in the HFIR. The final burnup was $4.43 \pm 0.31 \mathrm{GWd} / \mathrm{tHM}$ (Swinney 2015). The

95 irradiation produced $0.196 \pm 0.013 \mathrm{mg}$ of total $\mathrm{Pu}$ as measured by ICP-MS, with the assumption that, due to the low burnup, all of the 241 mass was ${ }^{241} \mathrm{Pu}$. After the short-lived radioisotopes had opportunity to decay, the irradiated pellet was shipped to Texas A\&M University.

The kerosene and TBP $(>99 \%)$ used for these experiments were acquired 100 from Alfa Aesar (Haverhill, Massachusetts, USA), $\mathrm{NaNO}_{2}$ was acquired from Aldrich Chemistry (St. Louis, Missouri, USA), 69\% nitric acid was acquired from Mallinckrodt Chemicals (St. Louis, Missouri, USA), and iron sulfamate (40.26\%) was acquired from Strem Chemicals (Newburyport, Massachusetts, USA). Chemicals were used as received without further purification. Degradation of TBP 105 due to radiolysis was not a significant concern because the activities were relatively low, most of the FPs stayed in the aqueous phase, and the organic phase was only in contact with the aqueous for a few minutes at a time. 
The pellet, both prior to dissolution and after, was counted with a Canberra HPGe detector model number CC4018 which was connected to a Canberra Lynx multichannel analyzer (Zakrzewski 2013, Canberra 2013a). Canberra's software package GENIE-2000 version 3.2.1 (Canberra 2013b) was used to collect spectra while the samples were inside a lead tomb. The same detector was used to count the various process solutions. Inductively coupled plasma mass spectrometry (ICPMS) was also conducted for aqueous samples using a PerkinElmer NexION 300X 115 quadrupole ICP-MS (PerkinElmer 2009).

Samples of the pellet were prepared as described below. The sample was added to a round-bottom flask and $5.0 \mathrm{ml}$ of $8 \mathrm{M} \mathrm{HNO}_{3}$ was added to the flask, which was heated to $50{ }^{\circ} \mathrm{C}$ with constant $100 \mathrm{rpm}$ stirring for $2 \mathrm{~h}$. This solution will be referred to as the "dissolution solution". The flask was connected to a cold trap with the help of Schlenk line. The FP gases such as $\mathrm{H}_{2}, \mathrm{CO}_{2}, \mathrm{Kr}, \mathrm{Br}_{2}, \mathrm{I}_{2}$ and $\mathrm{N}_{2} \mathrm{O}$ were captured in the cold trap using molecular sieves that were chilled by liquid nitrogen. In order to reduce the amount of activity per sample, $500 \mu \mathrm{l}$ from the dissolution solution was diluted to $5.0 \mathrm{ml}$ and the concentration was changed to $4 \mathrm{M} \mathrm{HNO}_{3}$. From this solution, referred to as the "stock solution", $0.5 \mathrm{ml}$ 125 aliquots, containing $\sim 1 \%$ of the pellet, were used in benchtop scale PUREX experiments described in the two subsections below. The total activity concentration of the stock solution was approximately $80 \mu \mathrm{Ci} / \mathrm{ml}$.

The "working" solution was prepared by transferring a $500 \mu \mathrm{l}$ aliquot of stock solution and $0.5 \mathrm{mg}$ of $\mathrm{NaNO}_{2}$ to a $15 \mathrm{ml}$ centrifuge tube. The tube was subsequently stirred and covered to retain the resulting $\mathrm{NO}_{2}$ gas. The solution was 
left overnight to ensure complete oxidation of $\mathrm{Pu}(\mathrm{III})$ to $\mathrm{Pu}(\mathrm{IV})$. Pu exists in several oxidation states at room temperature, and interconversion can be rapid. As a result, it is possible for $\mathrm{Pu}(\mathrm{IV})$ to convert to other oxidations states after using $\mathrm{NaNO}_{2}$. Regardless, the transfer of $\mathrm{Pu}(\mathrm{IV})$ to the organic phase reduces the 135 concentration of $\mathrm{Pu}(\mathrm{IV})$ in the aqueous phase. In order to restore the equilibrium, other oxidation states will convert to $\mathrm{Pu}(\mathrm{IV})$, which can then be further extracted. The end result is preferential formation of $\mathrm{Pu}(\mathrm{IV})$ in the aqueous phase. The high extraction yields discussed below suggest that production of $\mathrm{Pu}(\mathrm{IV})$ was dominant as expected. During extraction and back-extraction, both experiments had the

140 aqueous and organic phases mixed on a vortex mixer for $15 \mathrm{~min}$ at $1500 \mathrm{rpm}$, after which the two phases were allowed to settle and separate. The phases were then physically separated into two different vials via pipetting.

Each extraction and back-extraction mixed organic and aqueous solutions with unequal volumes. The solution being added always contained an extra $200 \mu \mathrm{l}$ to

145 reduce the chance of accidentally pipetting both phases. For example, if TBP were being added to the working solution described above, $700 \mu$ l would be added initially and $500 \mu \mathrm{l}$ removed. This excess volume will be referred to as hold-up volume in similar fashion to the differential extraction experiment described by Long (Long 1967). All results presented below have been corrected to those that

150 would be expected for equal volumes between aqueous and organic solutions during both extraction and back-extraction. Results are also corrected to zero holdup volume. 
Experiment 1 . The purpose of the first experiment was to quantify product recovery and DF values for a single stage extraction and back-extraction of Pu. U(VI) and

$155 \mathrm{Pu}(\mathrm{IV})$ were extracted and decontaminated by contacting the prepared working solution with a solution of $30 \mathrm{vol} \%$ TBP with a kerosene diluent. After mixing and separation of the two phases, $\mathrm{Pu}(\mathrm{IV})$ was reduced to $\mathrm{Pu}(\mathrm{III})$ and back-extracted by contacting the physically separated TBP solution with a solution of $0.024 \mathrm{M}$ ferrous [Fe(II)] sulfamate in $0.75 \mathrm{M} \mathrm{HNO}_{3}$. The influence of $\mathrm{Fe}^{2+}$ concentration on FP DFs was not studied but is expected to be small because its concentration is already multiple orders of magnitude greater than the FPs, and any extraction enhancement would be expected to be observed even at low concentrations. Additionally, the extraction of FPs would depend on their ability to form neutral species, but this does not happen with $\mathrm{Fe}^{2+}$. All aqueous solutions were analyzed with ICP-MS.

Experiment 2. The purpose of the second experiment was to recover a large fraction of Pu. Utilizing the results from the first experiment, it was determined that contacting the prepared working solution four times with TBP would extract over 90\% of the Pu. Therefore, this experiment had four extraction stages with the prepared working solution. The four TBP solutions were then collected into a single vial, and contacted three times with $0.024 \mathrm{M}$ ferrous sulfamate in a $4 \mathrm{M}$ $\mathrm{HNO}_{3}$ solution. These three contacts were then collected into a single vial.

In order to ensure minimal $\mathrm{U}$ back-extraction, the $\mathrm{HNO}_{3}$ concentration for this experiment was increased to $4 \mathrm{M}$ because higher concentrations reduce the 175 degree to which U is back-extracted (Stoller 1961). Three contacts of the ferrous 
sulfamate solution ensured nearly complete back-extraction of $\mathrm{Pu}$, while the higher nitric acid concentration minimized back-extraction of $\mathrm{U}$. The same solutions as described in the first experiment were analyzed with ICP-MS.

The four-contact extraction, three-contact back-extraction process described

180 above for the second experiment will be referred to as a cycle. The combined ferrous sulfamate solution containing back-extracted Pu underwent a second cycle to verify the repeatability of the process and for comparison with the first cycle. The ending solution of the first cycle Pu was "reset" with the addition of $0.5 \mathrm{mg}$ of $\mathrm{NaNO}_{2}$ to convert all $\mathrm{Pu}(\mathrm{III})$ to $\mathrm{Pu}(\mathrm{IV})$.

\section{Results}

The $\mathrm{U}$ and Pu recovery after back-extraction for the first and second experiments are shown in Table 1. The DCs for $\mathrm{U}$ and $\mathrm{Pu}$ were determined by analyzing the working solution both before and after extraction for experiment 1 . These were determined to be $26.7 \pm 2.9$ and $11.6 \pm 1.1$, for $\mathrm{U}$ and Pu respectively. Experiment 2 successfully recovered approximately 93\% of the original Pu with less than $1 \%$ of the original $\mathrm{U}$ remaining.

Table 1 Recoveries of $\mathrm{U}$ and $\mathrm{Pu}$ for the different experiments.

\begin{tabular}{|l|l|l|}
\hline & Pu Recovery & U Recovery \\
\hline Experiment 1 & $(83.4 \pm 9.5) \%$ & $(11.2 \pm 1.3) \%$ \\
\hline Experiment 2 Cycle 1 & $(99.7 \pm 4.2) \%$ & $(6.8 \pm 0.3) \%$ \\
\hline Experiment 2 Cycle 2 & $(93.0 \pm 4.6) \%$ & $(6.6 \pm 0.3) \%$ \\
\hline Overall Experiment 2 & $(92.7 \pm 6.0) \%$ & $(0.45 \pm 0.03) \%$ \\
\hline
\end{tabular}

In experiment 1 , approximately $10 \%$ of the $\mathrm{U}$ in the TBP phase was back-

195 extracted with a single contact of a solution of $0.024 \mathrm{M}$ iron sulfamate in $0.75 \mathrm{M}$ $\mathrm{HNO}_{3}$. Experiment 2 had much smaller $\mathrm{U}$ back-extraction due to the higher molar 
concentration of $\mathrm{HNO}_{3}$ in the back-extraction solution (Benedict 1982). This can potentially led to lower Pu recovery because the stability of ferrous ion decreases as the concentration of nitric acid increases (Stoller 1961). The half-life of the ferrous ion in the back-extraction solution of experiment 2 was estimated to be approximately $4 \mathrm{~d}$ (Stoller 1961). The effect is shown in the differences in $\mathrm{Pu}$ recovery between cycle 1 and cycle 2 of experiment 2 , where cycle 2 backextraction utilized a Fe(II) solution that was $1 \mathrm{~d}$ older. This effect was expected to be small for the FPs because of their strong preference for the aqueous phase (low 205 DCs).

The DF calculations utilized concentration ratios between contaminants that were normalized to the Pu concentration per Equation 3, with the initial solution being the working solution and the final solution being the back-extracted Pu. Both experiment 1 and experiment 2 first cycle DF values are shown in Table 2.

210 FPs of interest were selected based on our previous publication (Chirayath 2015) with additions of $\mathrm{Mo}, \mathrm{Ru}, \mathrm{Pd}, \mathrm{Cd}$, and Ce. ${ }^{237} \mathrm{~Np}$ and ${ }^{241} \mathrm{Am}$ were not considered due to their low forensic value in this case. ${ }^{99} \mathrm{Tc}$ was also not considered due to its low forensic value and difficulty of assaying the low-intensity photons produced by this nuclide. Cycle 2 of experiment 2 is not shown because the data were below 215 background for these samples, which was generally less than $1 \mathrm{ppb}$. The low DF values reported were expected due to the reasons described above: the extraction and back-extraction were performed without intermittent scrubbing, and the number of stages was low.

Table 2 Decontamination factors for single and multiple-contact PUREX.

\begin{tabular}{l|l|l|l|l|l} 
Element & Exp. 1 & Error & Exp. 2 Cycle 1 & Error & Isotopes
\end{tabular}




\begin{tabular}{|c|c|c|r|c|c|}
\hline $\mathbf{( Z )}$ & & & & & Used \\
\hline Rb (37) & 39.0 & 5.9 & 11.8 & 0.8 & ${ }^{85} \mathrm{Rb}$ \\
\hline Sr (38) & 283 & 43 & 84.6 & 5.9 & ${ }^{90} \mathrm{Sr}$ \\
\hline Mo (42) & 5.7 & 0.8 & 1.9 & 0.2 & ${ }^{97,98,100} \mathrm{Mo}$ \\
\hline Ru (44) & 59.2 & 6.4 & 16.6 & 2.5 & $101,102,104 \mathrm{Ru}$ \\
\hline Pd (46) & 65 & 14 & 8.9 & 1.2 & ${ }^{110} \mathrm{Pd}$ \\
\hline Cd (48) & 74 & 17 & 22.1 & 2.5 & ${ }^{112} \mathrm{Cd}$ \\
\hline Cs (55) & 177 & 28 & 52.9 & 3.9 & ${ }^{133} \mathrm{Cs}$ \\
\hline Ce (58) & 43 & 16 & 11.5 & 4.9 & ${ }^{140,142} \mathrm{Ce}$ \\
\hline Nd (60) & 19.2 & 2.1 & 5.9 & 0.4 & ${ }^{143} \mathrm{Nd}$ \\
\hline Pm (61) & 12.8 & 1.9 & 3.9 & 0.3 & ${ }^{147} \mathrm{Pm}$ \\
\hline Sm (62) & 11.5 & 1.5 & 3.6 & 0.3 & ${ }^{151} \mathrm{Sm}$ \\
\hline Eu (63) & 10.0 & 1.4 & 3.6 & 0.3 & ${ }^{154} \mathrm{Eu}$ \\
\hline U (92) & 7.4 & 1.2 & 14.7 & 0.9 & ${ }^{238} \mathrm{U}$ \\
\hline
\end{tabular}

The common trend is that DF decreases from experiment 1 to experiment 2. The major exception is $\mathrm{U}$, which has a higher DF value. This is expected due to the change in $\mathrm{HNO}_{3}$ concentration in the iron sulfamate solution, where higher concentrations of $\mathrm{HNO}_{3}$ reduce the degree of $\mathrm{U}$ back-extraction, as mentioned

225 above. The rest of the elements have lower DF values because of the four extraction and three back-extraction steps in cycle 1 of experiment 2 compared to one extraction and one back extraction in experiment 1. This is illustrated in Figure 1, where theoretical DFs as a function of extraction step and volume ratio for a product with a DC of 10 and a contaminant with a DC of 0.1 are shown. Higher numbers of contacts decrease DF because less and less product is extracted with each step, while the amount of contaminant extracted is approximately unchanged. Experiment $2 \mathrm{DF}$ values decrease relative to experiment $1 \mathrm{DF}$ values because of this. 


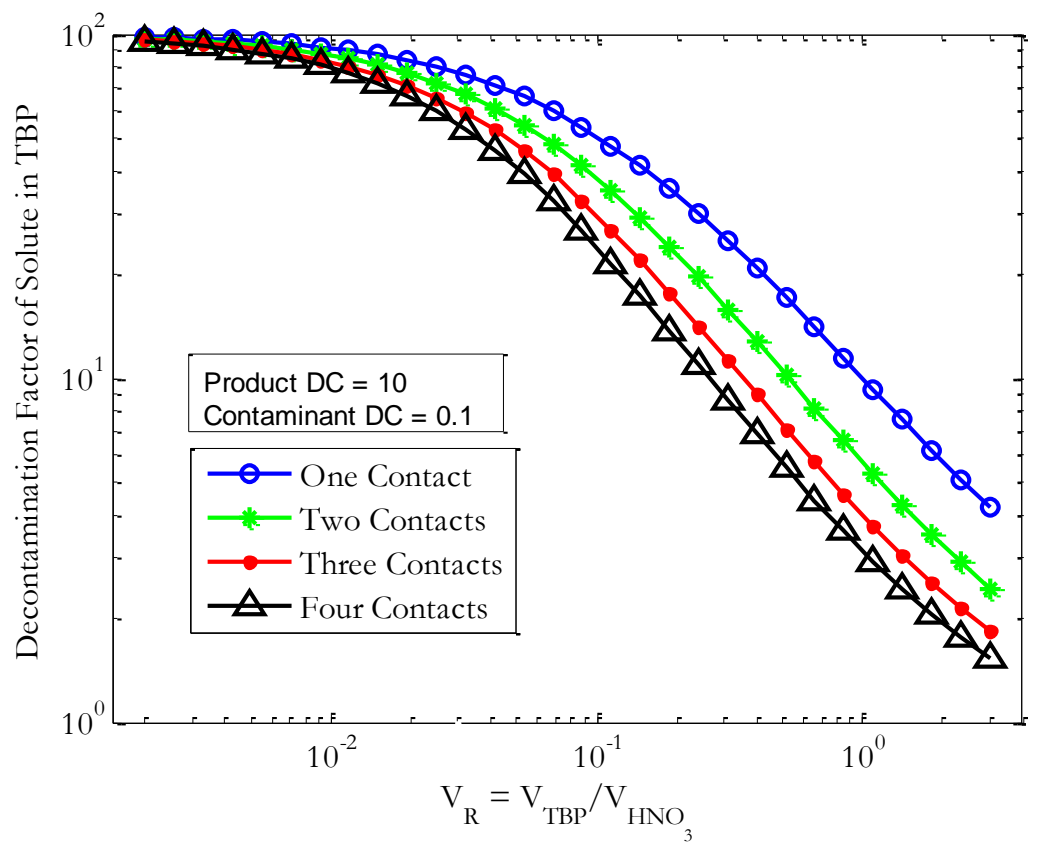

235 Figure $1 \mathrm{DFs}$ as a function of volume ratios for the first to fourth contact in TBP.

\section{Conclusions}

Two PUREX experiments were conducted. The first experiment determined

DC values for $\mathrm{Pu}, \mathrm{U}$ and several FPs. The second experiment utilized these values to recover over $92 \%$ of the $\mathrm{Pu}$ while leaving less than $1 \%$ of the $\mathrm{U}$. Overall $\mathrm{Pu}$ and U recoveries for experiment 1 were $(83.4 \pm 9.5) \%$ and $(11.2 \pm 1.3) \%$, respectively, while the same values for experiment 2 cycle 1 were $(99.7 \pm 4.2) \%$ and $(6.8 \pm$

$2450.3) \%$, respectively. DF values were measured for $12 \mathrm{FP}$ elements. DF values were lower than those typically found in industrial scale PUREX plants due to multiple 
extraction and back-extraction steps without an intermittent scrubbing step. This work provides critical DF data that will be needed for potential nuclear forensic investigations of interdicted Pu. Such a sample might have already been purified, so accurate DF data will allow for estimation of the original amounts of forensic isotopes. Regardless, additional research is needed to study the effects of additional scrubbing and stripping steps on individual DFs.

\section{Acknowledgements}

The work is supported by the U.S. Department of Homeland Security,

255 Domestic Nuclear Detection Office, through the U.S. National Science

Foundation under award numbers ECCS-1140018, 2012-DN-077-ARI1057-

02\&03, and 2015-DN-077-ARI099. The views and conclusions contained in this document are those of the authors and should not be interpreted as necessarily representing the official policies, either expressed or implied, of the U.S.

Department of Homeland Security.

\section{References}

Alcock, K., Bedford, F., Hardwick, W. and McKay, H. (1957). "Tri-n-butyl phosphate as an extracting solvent for inorganic nitrates-I: Zirconium

265 nitrate." Journal of Inorganic and Nuclear Chemistry 4(2): 100-105. phosphate as an extracting solvent for inorganic nitrates-V: Further results for the tetra-and hexavalent actinide nitrates." Journal of Inorganic and Nuclear Chemistry 6(4): 328-333.

270 Arker, A. J. (1954). "Terminal report on PUREX program in KAPL separations pilot plant." Knolls Atomic Power Laboratory.

Benedict, M., Levi, H. and Pigford, T. (1982). Nuclear chemical engineering, McGraw-Hill Pulishing. 84-214

Best, G., Hesford, E. and McKay, H. (1959). "Tri-n-butyl phosphate as an extracting agent for inorganic nitrates-VII: The trivalent actinide nitrates." Journal of Inorganic and Nuclear Chemistry 12(1): 136-140. 
Best, G., McKay, H. and Woodgate, P. (1957). "Tri-n-butyl phosphate as an extracting solvent for inorganic nitrates-III The plutonium nitrates." Journal of Inorganic and Nuclear Chemistry 4(5): 315-320.

Canberra. (2013a). "Standard electrode coaxial Ge detectors (SEGe)."

Retrieved 4/6/2016, from

http://www.canberra.com/products/detectors/pdf/SEGe-detectorsC40021.pdf.

Canberra. (2013b). "Genie 2000 basic spectroscopy software." Retrieved 4/6/2016, from http://www.canberra.com/products/radiochemistry lab/pdf/G2KBasicSpect-SS-C40220.pdf.

Chirayath, S. S., Osborn, J. M. and Coles, T. M. (2015). "Trace Fission Product Ratios for Nuclear Forensics Attribution of Weapons-Grade Plutonium from Fast and Thermal Reactors." Science \& Global Security 23(1): 48-67.

Colburn, A. P. (1939). "Simplified calculation of diffusional processes." general consideration of two-film resistances 35: 211-236.

Darby, D. O. and Chandler, J. M. (1954). "Terminal report for the ORNL pilot plant investigation of the PUREX process." Oak Ridge National Laboratory USAEC Report ORNL -1519.

Hesford, E., Jackson, E. and McKay, H. (1959). "Tri-n-butyl phosphate as an extracting agent for inorganic nitrates-VI Further results for the rare earth nitrates." Journal of Inorganic and Nuclear Chemistry 9(3-4):

300 279-289.

Hesford, E., McKay, H. and Scargill, D. (1957). "Tri-n-butyl phosphate as an extracting solvent for inorganic nitrates-IV Thorium nitrate." Journal of Inorganic and Nuclear Chemistry 4(5): 321-325.

Irish, E. R. and Reas, W. H. (1957). The PUREX process-a solvent extraction reprocessing method for irradiated uranium. Richland, Washington, Hanford Atomic Products Operation.

Lanham, W. B. and Gresky, A. T. (1950). "Purex process laboratory development." Oak Ridge National Laboratory USAEC Report ORNL-717.

Long, J. T. (1967). Engineering for nuclear fuel reprocessing, New York : Gordon and Breach Science Publishers. 162-272

PerkinElmer. (2009). "PerkinElmer NexION 300X quadrupole ICP-MS." Retrieved 4/6/2016, from https://partners.perkinelmer.com/Content/DealerSalesInfo/Product $\% 20$ Lines/Inorganic/ICPMS/Brochures/NexION\%20300\%20BRO DEF.pdf.

Perry, R. H. and Green, D. W. (2008). Perry's chemical engineers' handbook. New York, McGraw-Hill.

Scargill, D., Alcock, K., Fletcher, J., Hesford, E. and McKay, H. (1957). "Tri-nbutyl phosphate as an extracting solvent for inorganic nitrates-II 
Yttrium and the lower lanthanide nitrates." Journal of Inorganic and Nuclear Chemistry 4(5): 304-314.

Sherwood, T. K. and Pigford, R. L. (1952). Absorption and extraction. 2d ed, New York : McGraw-Hill.

325 Siddall, T. H., Prout, W. E., Parker, S. G., Commission, U. S. A. E., Savannah River, L., Nemours, E. I. d. P. d. and Company (1957). Equilibrium distribution data for purex and similar extraction processes, E.I. du Pont de Nemours \& Co., Explosives Dept., Atomic Energy Division, Technical Division, Savannah River Laboratory.

330 Simpson, F. M. and Law, D. J. (2010). Nuclear fuel reprocessing - INL/EXT10-17753, Idaho National Laboratory (INL).

Stoller, S. and Richards, R. (1961). "Reactor handbook, volume II, fuel reprocessing." Inter science Publishers, Inc., New York: 107-234.

Swinney, M. W. (2015). Experimental and computational assessment of trace nuclide ratios in weapons grade plutonium for nuclear forensics analysis. Doctor of Philosophy, Texas A\&M University.

Swinney, M. W. and Chirayath, S. S. (2014). "Comparison of FBR and HFIR Monte-Carlo Simulations with Validation from Gamma Spectroscopy in Support of the NFASP Project." Transactions of American Nuclear Society 111(1): 999-1001.

Zakrzewski, B. and Jordanov, V. (2013). "Versatility of modern digital signal processing: LYNX® - a platform for global spectroscopy applications." Digital Nuclear Spectroscopy: 87. 